\title{
Strength, anisotropy, and preferred orientation of solid argon at high pressures
}

\author{
Ho-kwang Mao ${ }^{1}$, James Badro ${ }^{2}$, Jinfu Shu ${ }^{1}$, Russell J Hemley ${ }^{1}$ and \\ Anil K Singh ${ }^{3}$ \\ ${ }^{1}$ Geophysical Laboratory, Carnegie Institution of Washington, 5251 Broad Branch Road, NW, \\ Washington, DC 20015, USA \\ ${ }^{2}$ Laboratoire de Minéralogie-Cristallographie, Institut de Physique du Globe de Paris, Université \\ Pierre et Marie Curie, 140 rue de Lourmel, 75015 Paris, France \\ ${ }^{3}$ Materials Science Division, National Aerospace Laboratories, Bangalore 560017, India \\ E-mail: mao@gl.ciw.edu
}

Received 7 May 2006

Published 8 June 2006

Online at stacks.iop.org/JPhysCM/18/S963

\begin{abstract}
The elasticity and plasticity of materials at high pressure are of great importance for the fundamental insight they provide on bonding properties in dense matter and for applications ranging from geophysics to materials technology. We studied pressure-solidified argon with a boron-epoxy-beryllium composite gasket in a diamond anvil cell (DAC). Employing monochromatic synchrotron $\mathrm{X}$-radiation and imaging plates in a radial diffraction geometry (Singh et al 1998 Phys. Rev. Lett. 80 2157; Mao et al 1998 Nature 396 741), we observed low strength in solid argon below $20 \mathrm{GPa}$, but the strength increases drastically with applied pressure, such that at $55 \mathrm{GPa}$, the shear strength exceeded $2.7 \mathrm{GPa}$. The elastic anisotropy at $55 \mathrm{GPa}$ was four times higher than the extrapolated value from $30 \mathrm{GPa}$. Extensive (111) slip develops under uniaxial compression, as manifested by the preferred crystallographic orientation of (220) in the compression direction. These macroscopic properties reflect basic changes in van der Waals bondings under ultrahigh pressures.
\end{abstract}

\section{Introduction}

The application of very high pressures can impart fundamental changes in the bonding characteristics of materials, thus altering their mechanical properties. The pressure effect on mechanical strength is still a poorly understood property which is difficult to predict theoretically [3] at high pressures. With the pressure gradient method [4] and the radial xray diffraction method $[2,5]$, the shear strength of solids can be measured in a DAC to hundreds of GPa. The shear strengths of most materials with metallic, ionic, and covalent bonding, such as $\mathrm{Fe}[6,7] \mathrm{Re}[8], \mathrm{NaCl}[9,10], \mathrm{MgO}$ [10-13], $\mathrm{Al}_{2} \mathrm{O}_{3}$ [14], $\mathrm{B}_{6} \mathrm{O}$ [15], pyrite [16], ringwoodite $[17,18]$, and perovskites $[19,20]$, increase linearly or sub-linearly 
with increasing pressures. Materials often soften at pressure-induced phase transformations, such as in the examples of coordination changes in amorphous $\mathrm{SiO}_{2}$ [21], stishovite- $\mathrm{CaCl}_{2}$ structural transition in $\mathrm{SiO}_{2}$ [22], and $\mathrm{B} 1-\mathrm{B} 2$ transition in $\mathrm{NaCl}$ [9], but can also harden drastically at a major bonding change, such as in the example of cold compressed graphite [23] and BN [24]. Noble gas solids with van der Waals-type bonding are often regarded as very weak, and therefore are used as quasi-hydrostatic pressure transmitting media in high-pressure experiments (for example, [25, 26]). Pressure-induced hardening of noble gas solids has been reported at high pressures $[27,28]$, but these studies have been limited to indirect observations of pressure gradient or the broadening of x-ray diffraction peaks of samples imbedded in the media. Here we report the quantitative determination of the strength of solid argon as a function of pressure up to $55 \mathrm{GPa}$ using radial x-ray diffraction in a DAC.

\section{Experiments}

To acquire the high resolution necessary for differentiating the small strain in argon, we employed the angular dispersive x-ray diffraction (ADXD) technique with $33.169 \mathrm{keV}$ monochromatic X-radiation (calibrated with the iodine K edge) with the Fastscan imaging plate system at the ID30 undulator beamline of the European Synchrotron Radiation Facility (ESRF). Complete diffraction rings were obtained from the same sample volume simultaneously, thus eliminating the uncertainties associated with pressure gradients and drift in energy-dispersive $\mathrm{x}$-ray diffraction studies [2]. The incident $\mathrm{x}$-ray is normal to the compression axis and passes through the $5 \mathrm{~mm}$ diameter of the $1 \mathrm{~mm}$ thick disc-shaped beryllium gasket. To avoid the background diffraction from the $5 \mathrm{~mm}$-thick Be gasket overwhelming the diffraction of the tiny $(60 \mu \mathrm{m})$ Ar sample, we drilled $200 \mu \mathrm{m}$-diameter holes from both sides of the Be gasket to remove $4.5 \mathrm{~mm}$ of the $5 \mathrm{~mm}$ Be along the x-ray beam path and refilled the holes with amorphous boron epoxy to maintain the support [19]. Diffraction rings from the remaining $500 \mu \mathrm{m} \mathrm{Be}$ in the X-ray path have comparable intensities to those of the $60 \mu \mathrm{m}$-deep Ar but do not overlap with the Ar rings (figure 1), thus serving as a useful reference. Diffraction patterns at 13, 28, 40, and $55 \mathrm{GPa}$ were collected and processed with the Fit2d code [29].

\section{Results}

The sample is subjected to two stress components in the DAC: axial $\left(\sigma_{3}\right)$ and radial $\left(\sigma_{1}\right)$ stresses. The average pressure is

$$
P=\left(\sigma_{3}+2 \sigma_{1}\right) / 3
$$

and the deviatoric stress is

$$
t=\sigma_{3}-\sigma_{1}
$$

which is also the lower bound of the shear strength. In the ADXD geometry, the azimuth angle $(\varphi)$ of the DAC, where the DAC compression axis is at $\varphi=0^{\circ}$ and $180^{\circ}$, is related to the azimuth angle $(\alpha)$ of an $\mathrm{x}$-ray diffraction ring with a $2 \theta$ diffraction angle, where the projection of the DAC compression axis on the imaging plate is at $\alpha=0^{\circ}$ and $180^{\circ}$, by

$$
\cos \varphi=\cos \theta \cos \alpha .
$$

Under the deviatoric stress, Ar shows a strong preferred orientation. Most notably, the (220) diffraction intensity is strongest at $\alpha=0^{\circ}$ and $180^{\circ}$ (the compression axis), and the (200) diffraction intensity shows maxima at $\alpha=45^{\circ}, 135^{\circ}, 225^{\circ}$ and $315^{\circ}$ (figure 1). This indicates that the van der Waals-bonded solid Ar deforms by (111) slip, similarly to metallic-bonded fcc 


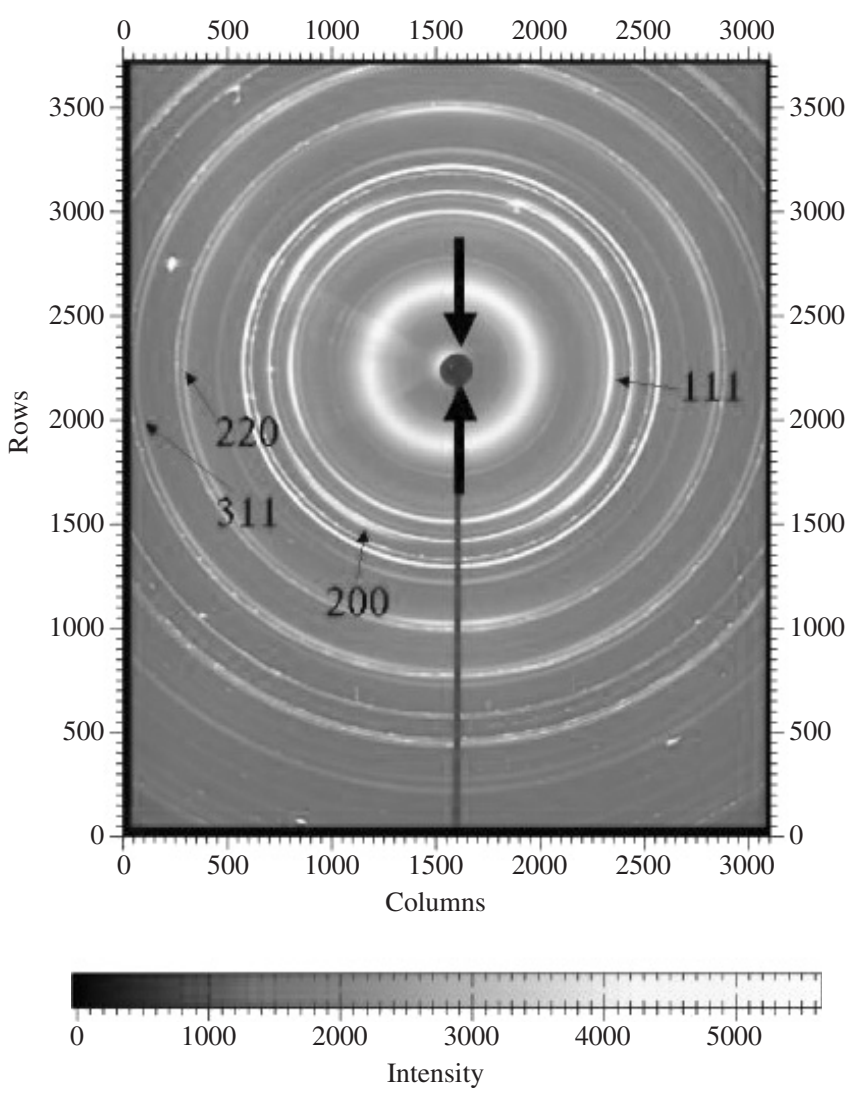

Figure 1. Diffraction pattern recorded by Fastscan imaging plate system at $55 \mathrm{GPa}$. The compression axis is shown as thick arrows. Four argon rings and their indices $(h k l)$ are labelled and indicated by arrows. All other rings are from the Be gasket or boron epoxy filler. The dark circle at the centre and the vertical line below are the shadow of the x-ray beam stop and the supporting wire at $\alpha=180^{\circ}$. The argon rings show strong ellipticity and preferred orientation. For instance, the $\mathrm{Ar}(220)$ ring is much closer to the circular Be ring immediately outside (the adjacent $\mathrm{Ar}$ and $\mathrm{Be}$ rings appearing as a doublet) at $\alpha=0^{\circ}$ and $180^{\circ}$ than at $90^{\circ}$ and $270^{\circ}$, indicating the elongation of the Ar ellipse toward $0^{\circ}$ and $180^{\circ}$ (the compression axis).

metals [30]. This also explains why Ar (200) and (220) reflections are often weak or absent in comparison to the (111) reflection when $\mathrm{Ar}$ is used as a pressure medium in routine axial x-ray diffraction experiments $\left(\alpha=90^{\circ} \pm 10^{\circ}\right)$ at the HPCAT, Sector 16 beamline of the Advanced Photon Sources, Argonne National Laboratory.

Below $20 \mathrm{GPa}$, Ar diffraction rings are nearly circular; at higher pressures, they become increasingly elliptical. At $55 \mathrm{GPa}$, the Ar rings are visibly elliptical in comparison to the neighbouring circular Be ring (figure 1). The observed ellipticity indicates a dramatic increase in the Ar strength. As shown in figure 2, the ellipse can be expressed as a trigonometric function of $d$-spacing and $\varphi$

$$
d=d_{\mathrm{P}}\left[1+\left(1-3 \cos ^{2} \varphi\right) Q\right]
$$

where the slope $Q$ indicates the magnitude of the differential strain [1]. The bulk shear strength of Ar can be obtained from

$$
t=6 G\langle Q\rangle
$$




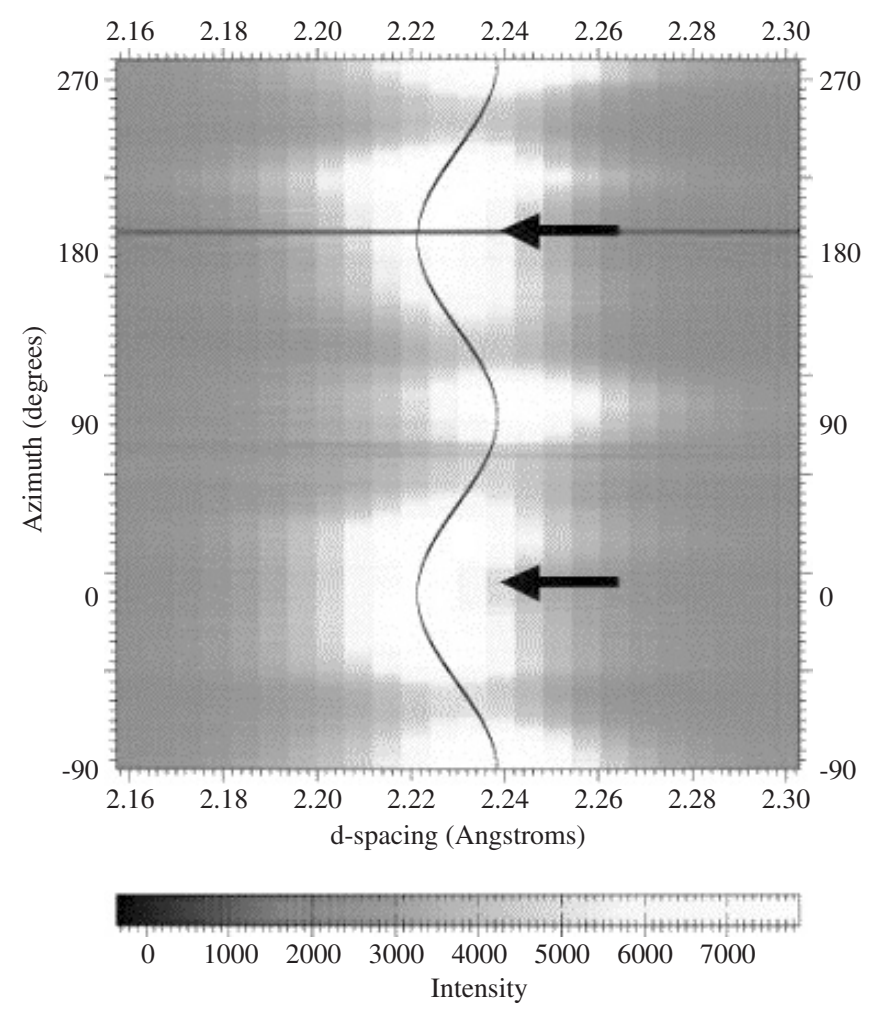

Figure 2. Diffraction ring of $\operatorname{Ar}(111)$ converted to Cartesian coordination of $d$-spacings versus azimuth angle $\alpha$. The compression directions at $0^{\circ}$ and $180^{\circ}$ are shown as thick arrows.

where $\langle Q\rangle$ denotes the average $Q(h k l)$, and $G$ is the shear modulus from the Brillouin data [31]. The values of $t$ are initially very low, but increase superlinearly with pressure (figure 3); the $\Delta t / \Delta P$ is 0.02 between 13 and $28 \mathrm{GPa}$, but increases to 0.08 between 40 and $55 \mathrm{GPa}$. The relation fits a parabolic function:

$$
t(\mathrm{GPa})=0.001 P^{2}-0.01 P .
$$

At $55 \mathrm{GPa}, t$ for Ar reaches $2.7 \mathrm{GPa}$, which exceeds the strength of hardened steel at ambient conditions. The astonishing superstrength of Ar above $30 \mathrm{GPa}$ reflects major changes in the van der Waals bonding.

The degree of ellipticity of the diffraction rings is also a function of the crystallographic orientation. As shown in figure 2, the $d$-spacing varies by as much as $0.7 \%$ between axial $\left(\alpha=0^{\circ}\right)$ and radial $\left(\alpha=90^{\circ}\right)$ directions for the $\operatorname{Ar}(111)$ plane, and $1.3 \%$ for $\operatorname{Ar}(200)$. For cubic crystals [1], the $h k l$ dependence of $Q$ can be expressed as a linear function of $B$ (figure 4), where $B=3\left(h^{2} k^{2}+k^{2} l^{2}+l^{2} h^{2}\right) /\left(h^{2}+k^{2}+l^{2}\right)^{2}$ denotes the orientation, and $A=\left(2 c_{44}\right)^{-1}-\left(c_{11}-c_{12}\right)^{-1}$ denotes the elastic anisotropy.

$$
\begin{aligned}
& Q=m_{0}+m_{1} B \\
& 3 m_{1}=t\left[\left(2 c_{44}\right)^{-1}-\left(c_{11}-c_{12}\right)^{-1}\right] .
\end{aligned}
$$

The observed $m_{1}=-2 \times 10^{-3}$ at $55 \mathrm{GPa}$ is considerably larger than $m_{1}=-5 \times 10^{-4}$ calculated using extrapolated $c_{i j}$ values from Brillouin spectroscopy measurements up to $30 \mathrm{GPa}$ [31], indicating significant changes of bonding at high pressures. 


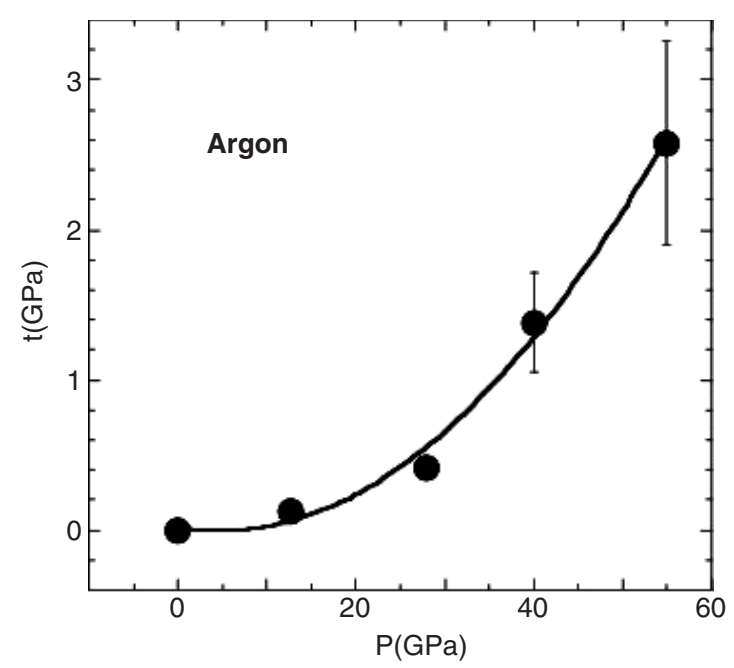

Figure 3. Deviatoric stress, which is the lower bound of the strength, of Ar as a function of pressure. The vertical bars indicate the range at different crystallographic orientation $(h k l)$. The true error bars are significantly smaller.

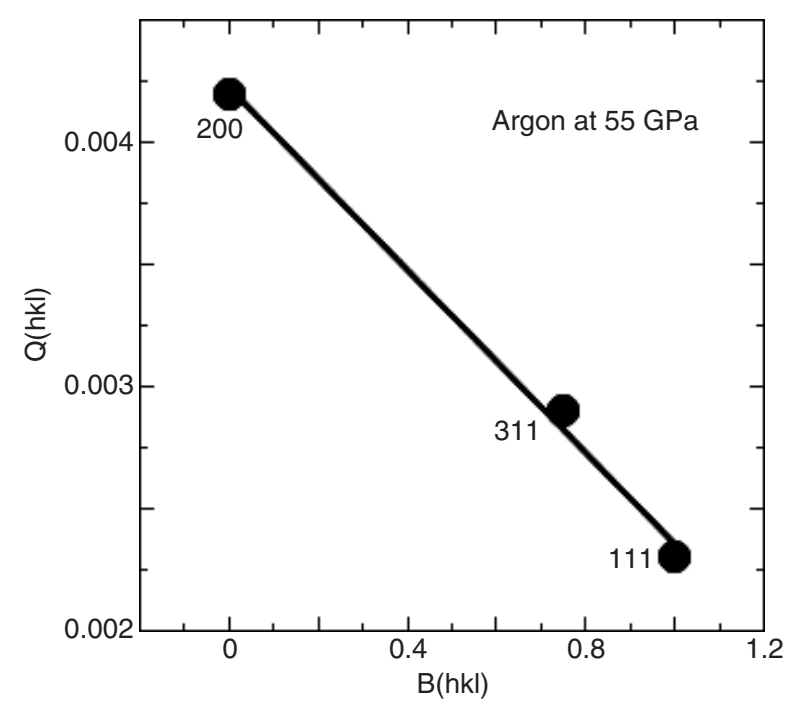

Figure 4. Differential strain of Ar at $55 \mathrm{GPa}$ as a function of $B(h k l)$.

\section{Conclusion}

In conclusion, the present observations of pressure-induced strength and elastic anisotropy in solid argon reveal drastic changes of van der Waals bondings that dictate the structure and chemistry of noble gas elements at high pressures. For instance, the melting temperatures of argon and xenon increase steeply with pressure and exceed that of iron at $30 \mathrm{GPa}$ [32]. The present observations provide valuable constraints on the theoretical understanding of the behaviour of simple solids at high densities, including the evolution of many-body effects with compression [33, 34]. 


\section{Acknowledgments}

We thank Haozhe Liu and Hans-Rudolf Wenk for valuable comments and Tristan LeBihan for help at the synchrotron beamline. The synchrotron facilities are supported by the ESRF. Support was provided by National Science Foundation-Earth Sciences (NSF-EAR), Department of Energy-Basic Energy Sciences (DOE-BES) (HPCAT), DOE-National Nuclear Security Administration (NNSA) (Carnegie/DOE Alliance Center), and the W M Keck Foundation of the United States and CNRS of France. Laboratoire de MinéralogieCristallographie is CNRS UMR 7590.

\section{References}

[1] Singh A K, Mao H K, Shu J and Hemley R J 1998 Phys. Rev. Lett. 802157

[2] Mao H K, Shu J, Shen G, Hemley R J, Li B and Singh A K 1998 Nature 396741

[3] Söderlind P, Yang L H, Moriarty J A and Wills J M 2000 Phys. Rev. B 612579

[4] Sung C-M, Goetze C and Mao H K 1977 Rev. Sci. Instrum. 481386

[5] Kinsland G L and Bassett W A 1976 Rev. Sci. Instrum. 47130

[6] Hemley R J, Mao H K, Shen G, Badro J, Gillet P, Hanfland M and Häusermann D 1997 Science 2761242

[7] Merkel S, Shu J, Gillet P, Mao H K and Hemley R J 2005 J. Geophys. Res. 110 B025201

[8] Jeanloz R, Godwal B K and Meade C 1991 Nature 349687

[9] Meade C and Jeanloz R 1988 J. Geophys. Res. 933270

[10] Kinsland G L and Bassett W A 1977 J. Appl. Phys. 48978

[11] Meade C and Jeanloz R 1988 J. Geophys. Res. 933261

[12] Duffy T S, Hemley R J and Mao H K 1995 Phys. Rev. Lett. 741371

[13] Merkel S, Wenk H R, Shu J, Shen G, Gillet P, Mao H K and Hemley R J 2002 J. Geophys. Res. 107 doi:10.1029/2001JB000920

[14] Meade C and Jeanloz R 1990 Phys. Rev. B 422532

[15] He D, Shieh S R and Duffy T S 2004 Phys. Rev. B 70184121

[16] Merkel S, Jephcoat A P, Shu J, Mao H K, Gillet P and Hemley R J 2002 Phys. Chem. Miner. 291

[17] Kavner A and Duffy T S 2001 Geophys. Res. Lett. 282691

[18] Kavner A 2003 Earth Planet. Sci. Lett. 214645

[19] Merkel S, Wenk H R, Badro J, Montagnac G, Gillet P, Mao H K and Hemley R J 2003 Earth Planet. Sci. Lett. 209351

[20] Shieh S R, Duffy T and Shen G 2004 Phys. Earth Planet. Inter. 14393

[21] Meade C and Jeanloz R 1988 Science 2411072

[22] Shieh S R, Duffy T S and Li B 2002 Phys. Rev. Lett. 89255507

[23] Mao W L, Mao H K, Eng P, Trainor T, Newville M, Kao C C, Heinz D L, Shu J, Meng Y and Hemley R J 2003 Science $\mathbf{3 0 2} 425$

[24] Meng Y, Mao H K, Eng P, Trainor T, Newville M, Hu M Y, Kao C C, Häusermann D and Hemley R J 2004 Nat. Mater. 3111

[25] Errandonea D, Boehler R and Ross M 2002 Phys. Rev. B 65012108

[26] Chudinovskikh L and Boehler R 2001 Nature 411574

[27] Bell P M and Mao H K 1981 Carnegie Inst. Washington Yearb. 80404

[28] Takemura K 2001 J. Appl. Phys. 89662

[29] Hammersley J 1996 ESRF Report No. ESRF98HAO1T

[30] Wenk H-R, Lonardelli I, Pehl J, Devine J, Prakapenka V, Shen G and Mao H K 2004 Earth Planet. Sci. Lett. 226507

[31] Grimsditch M, Loubeyre P and Polian A 1986 Phys. Rev. B 337192

[32] Jephcoat A P 1998 Nature 393355

[33] Datchi F, Loubeyre P and LeToullec R 2000 Phys. Rev. B 616535

[34] Occelli F, Krisch M, Loubeyre P, Sette F, Toullec R L, Masciovecchio C and Rueff J-P 2001 Phys. Rev. B 63224306 